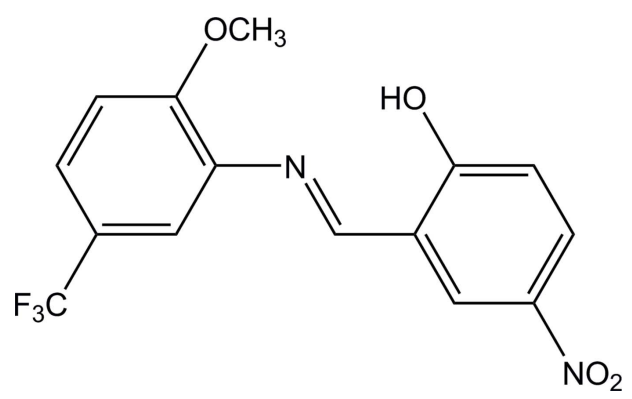

\section{Crystal structure of 2-\{[2-methoxy-5-(tri- fluoromethyl)phenyl]iminomethyl\}-4- nitrophenol}

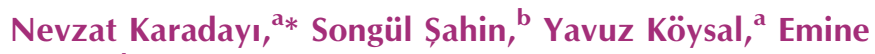
Coșkun $^{\mathrm{b}}$ and Orhan Büyükgüngör ${ }^{\mathrm{c}}$

${ }^{a}$ Yeşilyurt Demir Celik Higher Vocational School, Ondokuz Mayıs University, TR-55330 Tekkeköy-Samsun, Turkey, ${ }^{\mathbf{b}}$ Department of Chemistry, Faculty of Arts and Sciences, Ondokuz Mayıs University, TR-55139 Samsun, Turkey, and ${ }^{\mathbf{c}}$ Department of Physics, Faculty of Arts and Sciences, Ondokuz Mayıs University, TR-55139 Samsun, Turkey. *Correspondence e-mail: nevzatk@omu.edu.tr

Received 16 April 2015; accepted 25 May 2015

Edited by H. Ishida, Okayama University, Japan

In the title compound, $\mathrm{C}_{15} \mathrm{H}_{11} \mathrm{~F}_{3} \mathrm{~N}_{2} \mathrm{O}_{4}$, the $\mathrm{N}=\mathrm{C}$ bond of the central imine group adopts an $E$ conformation. The dihedral angle between two benzene rings is $6.2(2)^{\circ}$. There is an intramolecular bifurcated $\mathrm{O}-\mathrm{H} \cdots(\mathrm{N}, \mathrm{O})$ hydrogen bond with $S(6)$ and $S(9)$ ring motifs. In the crystal, molecules are linked by $\mathrm{C}-\mathrm{H} \cdots \mathrm{O}$ hydrogen bonds into a helical chain along the $3_{1}$ screw axis parallel to $c$. The $-\mathrm{CF}_{3}$ group shows rotational disorder over two sites, with occupancies of 0.39 (2) and $0.61(2)$.

Keywords: crystal structure; Schiff base; hydrogen bonding.

CCDC reference: 1402674

\section{Related literature}

For photochromic, thermochromic and biological applications of related Schiff base compounds, see: Hadjoudis et al. (1987); Santos et al. (2001); Tarafder et al. (2002). For related structures, see: Faridbod et al. (2008); Karadayı et al. (2003, 2006, 2013); Raja et al. (2008).

\section{Experimental}

2.1. Crystal data
$\mathrm{C}_{15} \mathrm{H}_{11} \mathrm{~F}_{3} \mathrm{~N}_{2} \mathrm{O}_{4}$

$M_{r}=340.26$

Trigonal, $R \overline{3}$

$c=7.1523(3) \AA$

$V=6758.7(5) \AA^{3}$

\subsection{Data collection}

Stoe IPDS 2 diffractometer Absorption correction: integration ( $X$-RED32; Stoe \& Cie, 2002)

$T_{\min }=0.951, T_{\max }=0.994$

\subsection{Refinement}

$R\left[F^{2}>2 \sigma\left(F^{2}\right)\right]=0.084$

$w R\left(F^{2}\right)=0.126$

$S=1.07$

2958 reflections $a=33.0327(16) \AA$

$$
\begin{aligned}
& Z=18 \\
& \text { Mo } K \alpha \text { radiation } \\
& \mu=0.13 \mathrm{~mm}^{-1} \\
& T=296 \mathrm{~K} \\
& 0.67 \times 0.25 \times 0.04 \mathrm{~mm}
\end{aligned}
$$

16356 measured reflections 2958 independent reflections 1380 reflections with $I>2 \sigma(I)$ $R_{\text {int }}=0.140$
Table 1

Hydrogen-bond geometry $\left(\AA,^{\circ}\right)$.

\begin{tabular}{lllll}
\hline$D-\mathrm{H} \cdots A$ & $D-\mathrm{H}$ & $\mathrm{H} \cdots A$ & $D \cdots A$ & $D-\mathrm{H} \cdots A$ \\
\hline $\mathrm{O} 1-\mathrm{H} 1 \cdots \mathrm{N} 1$ & 0.82 & 1.84 & $2.571(4)$ & 148 \\
$\mathrm{O} 1-\mathrm{H} 1 \cdots \mathrm{O} 4$ & 0.82 & 2.76 & $3.468(4)$ & 146 \\
$\mathrm{C} 7-\mathrm{H} 7 \cdots 2^{\mathrm{i}}$ & 0.93 & 2.55 & $3.476(7)$ & 176 \\
$\mathrm{C} 9-\mathrm{H} 9 \cdots 2^{\mathrm{i}}$ & 0.93 & 2.46 & $3.378(6)$ & 169
\end{tabular}

Symmetry code: (i) $-y+\frac{4}{3}, x-y-\frac{1}{3}, z-\frac{1}{3}$.

Data collection: $X$-AREA (Stoe \& Cie, 2002); cell refinement: $X$ $A R E A$; data reduction: $X$-RED32 (Stoe \& Cie, 2002); $\operatorname{program}(\mathrm{s})$ used to solve structure: SHELXS97 (Sheldrick, 2008); program(s) used to refine structure: SHELXL97 (Sheldrick, 2008); molecular graphics: ORTEP-3 for Windows (Farrugia, 2012); software used to prepare material for publication: Win $G X$ (Farrugia, 2012).

\section{Acknowledgements}

The authors wish to acknowledge the Faculty of Arts and Sciences, Ondokuz Mayıs University, Turkey, for the use of the Stoe IPDS II diffractometer (purchased under grant F.279 of the University Research Fund). 
Supporting information for this paper is available from the IUCr electronic archives (Reference: IS5398).

\section{References}

Faridbod, F., Ganjali, M. R., Dinarvand, R., Norouzi, P. \& Riahi, S. (2008). Sensors, 8, 1645-1703.

Farrugia, L. J. (2012). J. Appl. Cryst. 45, 849-854.

Hadjoudis, E., Vittorakis, M. \& Moustakali-Mavridis, I. (1987). Tetrahedron, $\mathbf{4 3}, 1345-1360$.

Karadayı, N., Albayrak, C., Odabaşoğlu, M. \& Büyükgüngör, O. (2006). Acta Cryst. E62, o1699-o1701.
Karadayı, N., Gözüyeşil, S., Güzel, B., Kazak, C. \& Büyükgüngör, O. (2003). Acta Cryst. E59, o851-0853.

Karadayı, N., Köysal, Y., Şahin, S., Coşkun, E. \& Büyükgüngör, O. (2013). Acta Cryst. E69, o889.

Raja, K. K., Bilal, I. M., Thambidurai, S., Rajagopal, G. \& SubbiahPandi, A. (2008). Acta Cryst. E64, o2265.

Santos, M. L. P., Bagatin, I. A., Pereira, E. M. \& Ferreira, A. M. D. C. (2001). J. Chem. Soc. Dalton Trans. pp. 838-844.

Sheldrick, G. M. (2008). Acta Cryst. A64, 112-122.

Stoe \& Cie (2002). $X$-AREA and $X$-RED32. Stoe \& Cie, Darmstadt, Germany. Tarafder, M. T. H., Chew, K., Crouse, K. A., Ali, A. M., Yamin, B. M. \& Fun, H.-K. (2002). Polyhedron, 21, 2683-2690. 


\section{supporting information}

Acta Cryst. (2015). E71, o466-o467 [doi:10.1107/S2056989015010129]

\section{Crystal structure of 2-\{[2-methoxy-5-(trifluoromethyl)phenyl]iminomethyl\}-4- nitrophenol}

\section{Nevzat Karadayı, Songül Şahin, Yavuz Köysal, Emine Coşkun and Orhan Büyükgüngör}

\section{S1. Comment}

Schiff base compounds can be classified by their photochromic and thermochromic characteristics (Hadjoudis et al., 1987). Schiff bases are potentially biologically active compounds and the antifungal, anticancer, anticonvulsant, diuretic and cytotoxic activities have been reported. For the development of bacteriostatic activity, it is believed that the presence of a nitro group in the $p$-position is an important condition (Tarafder et al., 2002; Santos et al., 2001). In this study we report the structure of the title compound (I).

The $\mathrm{N} 1=\mathrm{C} 7$ bond length is 1.295 (5) $\AA$, approximately equal to previously reported $\mathrm{C}=\mathrm{N}$ bond lengths (Karaday1 et al., 2003; Faridbod et al., 2008; Karaday1 et al., 2013). The geometric parameters in (I) are comparable with the similar reported structures (Raja et al., 2008; Karaday1 et al., 2006). The dihedral angle between the aromatic rings (C1-C6) and (C8-C13) is $6.2(2)^{\circ}$. The $\mathrm{CF}_{3}$ group showed rotational disorder. The site occupancy factors are 0.39 (2) and 0.61 (2) for F1A-F3A and F1B-F3B, respectively. An intramolecular bifurcated $\mathrm{O}-\mathrm{H} \cdots(\mathrm{N}, \mathrm{O})$ hydrogen bond is observed (Table 1 and Fig. 1).

\section{S2. Experimental}

The title compound was prepared by refluxing a mixture of a solution containing 2-hydroxy-5-nitrobenzaldehyde $(0.014$ g, $0.082 \mathrm{mmol}$ ) and a solution containing 2-methoxy-5-(trifluoromethyl)aniline (0,016 g, $0.082 \mathrm{mmol})$ in $20 \mathrm{ml}$ ethanol. The reaction mixture was stirred for $1 \mathrm{~h}$ under reflux. Single crystals suitable for X-ray analysis were obtained from an ethanol solution by slow evaporation (yield 54\%, m.p. 475-477 K).

\section{S3. Refinement}

All $\mathrm{H}$ atoms were placed in calculated positions and refined using a riding model, with $\mathrm{C}-\mathrm{H}=0.93$ or $0.96 \AA$ and $\mathrm{O}-\mathrm{H}$ $=0.82 \AA$. The isotropic displacement parameters of the $\mathrm{H}$ atoms were fixed at $1.2 U_{\mathrm{eq}}(\mathrm{C})$ or $1.5 U_{\mathrm{eq}}\left(\mathrm{O}, \mathrm{C}_{\text {methyl }}\right)$. The $\mathrm{CF}_{3}$ group showed rotational disorder. The site occupancy factors are 0.39 (2) and 0.61 (2) for F1A-F3A and F1B-F3B, respectively. 


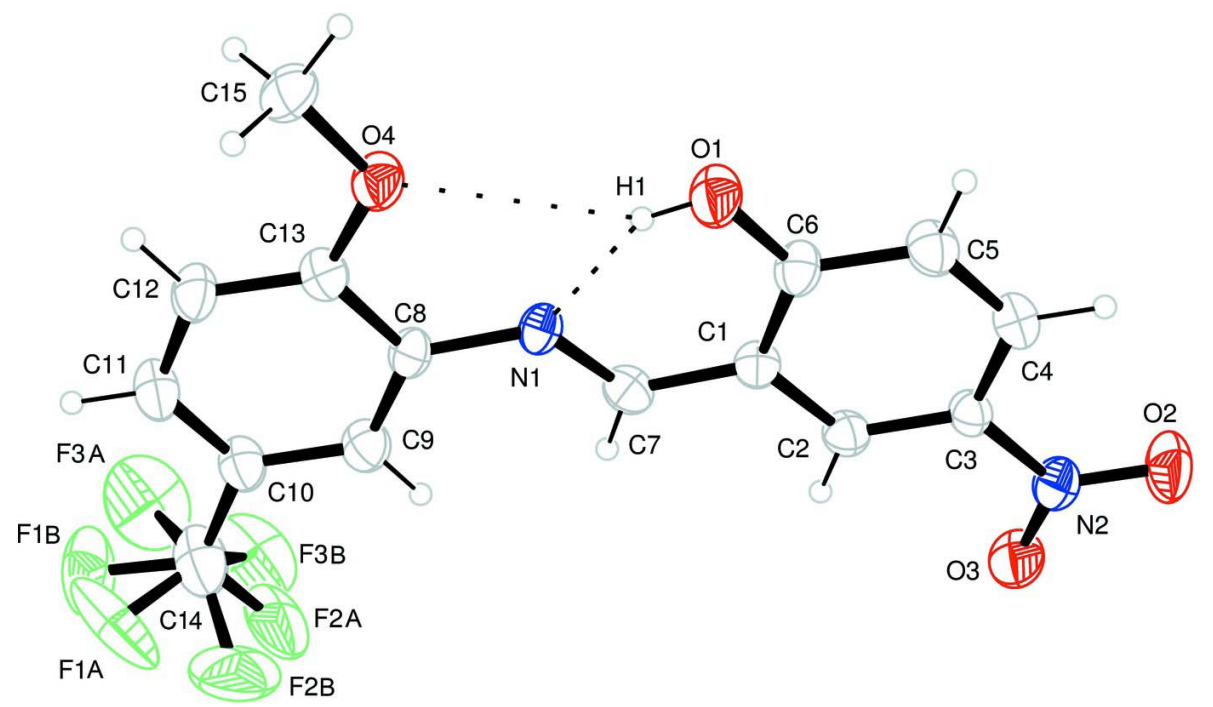

\section{Figure 1}

An ORTEP drawing of the title compound showing the atomic numbering scheme. Displacement ellipsoids of non- $\mathrm{H}$ atoms are shown at the $20 \%$ probability level. Hydrogen bonds are indicated by dashed lines.

\section{2-\{[2-Methoxy-5-(trifluoromethyl)phenyl]iminomethyl\}-4-nitrophenol}

\section{Crystal data}

$\mathrm{C}_{15} \mathrm{H}_{11} \mathrm{~F}_{3} \mathrm{~N}_{2} \mathrm{O}_{4}$

$M_{r}=340.26$

Trigonal, $R \overline{3}$

Hall symbol: $-\mathrm{R} 3$

$a=33.0327$ (16) $\AA$

$c=7.1523(3) \AA$

$V=6758.7(5) \AA^{3}$

$Z=18$

$F(000)=3132$

\section{Data collection}

Stoe IPDS 2

diffractometer

Radiation source: fine-focus sealed tube

Graphite monochromator

$\omega$ scans

Absorption correction: integration

(X-RED32; Stoe \& Cie, 2002)

$T_{\min }=0.951, T_{\max }=0.994$

\section{Refinement}

Refinement on $F^{2}$

Least-squares matrix: full

$R\left[F^{2}>2 \sigma\left(F^{2}\right)\right]=0.084$

$w R\left(F^{2}\right)=0.126$

$S=1.07$

2958 reflections

246 parameters

0 restraints
$D_{\mathrm{x}}=1.505 \mathrm{Mg} \mathrm{m}^{-3}$

Mo $K \alpha$ radiation, $\lambda=0.71073 \AA$

Cell parameters from 10608 reflections

$\theta=2.1-28.0^{\circ}$

$\mu=0.13 \mathrm{~mm}^{-1}$

$T=296 \mathrm{~K}$

Needle, light brown

$0.67 \times 0.25 \times 0.04 \mathrm{~mm}$

16356 measured reflections

2958 independent reflections

1380 reflections with $I>2 \sigma(I)$

$R_{\text {int }}=0.140$

$\theta_{\text {max }}=26.0^{\circ}, \theta_{\min }=2.1^{\circ}$

$h=-40 \rightarrow 40$

$k=-39 \rightarrow 40$

$l=-8 \rightarrow 8$

Primary atom site location: structure-invariant direct methods

Secondary atom site location: difference Fourier map

Hydrogen site location: inferred from neighbouring sites

$\mathrm{H}$-atom parameters constrained 
$w=1 /\left[\sigma^{2}\left(F_{\mathrm{o}}^{2}\right)+(0.0292 P)^{2}\right]$

where $P=\left(F_{\mathrm{o}}^{2}+2 F_{\mathrm{c}}^{2}\right) / 3$

$(\Delta / \sigma)_{\max }=0.001$

$$
\Delta \rho_{\max }=0.15 \mathrm{e} \AA^{-3}
$$$$
\Delta \rho_{\min }=-0.19 \mathrm{e} \AA^{-3}
$$

\section{Special details}

Geometry. All e.s.d.'s (except the e.s.d. in the dihedral angle between two 1.s. planes) are estimated using the full covariance matrix. The cell e.s.d.'s are taken into account individually in the estimation of e.s.d.'s in distances, angles and torsion angles; correlations between e.s.d.'s in cell parameters are only used when they are defined by crystal symmetry. An approximate (isotropic) treatment of cell e.s.d.'s is used for estimating e.s.d.'s involving 1.s. planes.

Refinement. Refinement of $F^{2}$ against ALL reflections. The weighted $R$-factor $w R$ and goodness of fit $S$ are based on $F^{2}$, conventional $R$-factors $R$ are based on $F$, with $F$ set to zero for negative $F^{2}$. The threshold expression of $F^{2}>\sigma\left(F^{2}\right)$ is used only for calculating $R$-factors(gt) etc. and is not relevant to the choice of reflections for refinement. $R$-factors based on $F^{2}$ are statistically about twice as large as those based on $F$, and $R$ - factors based on ALL data will be even larger.

Fractional atomic coordinates and isotropic or equivalent isotropic displacement parameters $\left(\AA^{2}\right)$

\begin{tabular}{|c|c|c|c|c|c|}
\hline & $x$ & $y$ & $z$ & $U_{\text {iso }} * / U_{\text {eq }}$ & Occ. $(<1)$ \\
\hline $\mathrm{O} 4$ & $0.76299(10)$ & $0.04667(9)$ & $0.2585(4)$ & $0.0569(8)$ & \\
\hline $\mathrm{O} 1$ & $0.76808(9)$ & $0.14868(10)$ & $0.4121(5)$ & $0.0647(9)$ & \\
\hline H1 & 0.7798 & 0.1330 & 0.3798 & $0.097^{*}$ & \\
\hline N1 & $0.83251(11)$ & $0.12822(11)$ & $0.3519(4)$ & $0.0443(8)$ & \\
\hline $\mathrm{N} 2$ & $0.90475(14)$ & $0.32508(12)$ & $0.6373(5)$ & $0.0538(9)$ & \\
\hline $\mathrm{C} 7$ & $0.86313(14)$ & $0.17045(14)$ & $0.4002(5)$ & $0.0473(10)$ & \\
\hline $\mathrm{H7}$ & 0.8947 & 0.1797 & 0.3969 & $0.057 *$ & \\
\hline $\mathrm{C} 1$ & $0.84911(13)$ & $0.20297(13)$ & $0.4583(5)$ & $0.0417(10)$ & \\
\hline $\mathrm{O} 3$ & $0.94606(11)$ & $0.33571(11)$ & $0.6341(5)$ & $0.0724(10)$ & \\
\hline C6 & $0.80087(14)$ & $0.18987(14)$ & $0.4640(6)$ & $0.0488(11)$ & \\
\hline $\mathrm{C} 4$ & $0.82253(15)$ & $0.26645(15)$ & $0.5825(6)$ & $0.0529(11)$ & \\
\hline $\mathrm{H} 4$ & 0.8141 & 0.2879 & 0.6241 & $0.063^{*}$ & \\
\hline $\mathrm{C} 2$ & $0.88280(14)$ & $0.24797(13)$ & $0.5163(6)$ & $0.0452(10)$ & \\
\hline $\mathrm{H} 2$ & 0.9143 & 0.2568 & 0.5131 & $0.054^{*}$ & \\
\hline $\mathrm{C} 3$ & $0.86965(13)$ & $0.27894(13)$ & $0.5775(6)$ & $0.0429(10)$ & \\
\hline C13 & $0.80545(15)$ & $0.04950(14)$ & $0.2514(6)$ & $0.0463(10)$ & \\
\hline $\mathrm{C} 10$ & $0.89519(15)$ & $0.06269(15)$ & $0.2580(6)$ & $0.0549(12)$ & \\
\hline $\mathrm{O} 2$ & $0.89212(12)$ & $0.35192(11)$ & $0.6969(5)$ & $0.0788(10)$ & \\
\hline $\mathrm{C} 11$ & $0.85770(17)$ & $0.01995(16)$ & $0.2083(6)$ & $0.0614(13)$ & \\
\hline H11 & 0.8627 & -0.0045 & 0.1772 & $0.074 *$ & \\
\hline $\mathrm{C} 5$ & $0.78900(15)$ & $0.22311(14)$ & $0.5269(6)$ & $0.0544(11)$ & \\
\hline $\mathrm{H} 5$ & 0.7578 & 0.2152 & 0.5303 & $0.065^{*}$ & \\
\hline C9 & $0.88791(15)$ & $0.09902(15)$ & $0.3038(6)$ & $0.0532(11)$ & \\
\hline $\mathrm{H} 9$ & 0.9132 & 0.1278 & 0.3373 & $0.064 *$ & \\
\hline $\mathrm{C} 8$ & $0.84359(13)$ & $0.09318(13)$ & $0.3005(5)$ & $0.0424(10)$ & \\
\hline $\mathrm{C} 12$ & $0.81262(16)$ & $0.01297(14)$ & $0.2039(6)$ & $0.0568(12)$ & \\
\hline $\mathrm{H} 12$ & 0.7876 & -0.0159 & 0.1695 & $0.068^{*}$ & \\
\hline $\mathrm{C} 15$ & $0.72262(15)$ & $0.00229(14)$ & $0.2147(6)$ & $0.0630(13)$ & \\
\hline $\mathrm{H} 15 \mathrm{~A}$ & 0.6950 & 0.0048 & 0.2240 & $0.094^{*}$ & \\
\hline H15B & 0.7205 & -0.0209 & 0.3011 & $0.094^{*}$ & \\
\hline $\mathrm{H} 15 \mathrm{C}$ & 0.7254 & -0.0066 & 0.0897 & $0.094 *$ & \\
\hline $\mathrm{C} 14$ & $0.9427(2)$ & $0.0689(2)$ & $0.2677(12)$ & $0.0853(18)$ & \\
\hline
\end{tabular}




\begin{tabular}{llllll} 
F1A & $0.9456(8)$ & $0.0369(9)$ & $0.340(5)$ & $0.142(14)$ & $0.39(2)$ \\
F2A & $0.9748(7)$ & $0.1072(7)$ & $0.317(7)$ & $0.158(16)$ & $0.39(2)$ \\
F3A & $0.9570(6)$ & $0.0665(12)$ & $0.074(2)$ & $0.152(9)$ & $0.39(2)$ \\
F1B & $0.9474(5)$ & $0.0345(4)$ & $0.221(3)$ & $0.136(9)$ & $0.61(2)$ \\
F2B & $0.9590(5)$ & $0.0783(9)$ & $0.4489(18)$ & $0.156(7)$ & $0.61(2)$ \\
F3B & $0.9727(4)$ & $0.1064(6)$ & $0.181(2)$ & $0.128(7)$ & $0.61(2)$ \\
\hline
\end{tabular}

Atomic displacement parameters $\left(\AA^{2}\right)$

\begin{tabular}{lllllll}
\hline & $U^{11}$ & $U^{22}$ & $U^{33}$ & $U^{12}$ & $U^{13}$ & $U^{23}$ \\
\hline O4 & $0.0435(17)$ & $0.0424(17)$ & $0.077(2)$ & $0.0155(15)$ & $0.0007(15)$ & $-0.0058(15)$ \\
O1 & $0.0437(17)$ & $0.048(2)$ & $0.097(3)$ & $0.0190(16)$ & $0.0001(17)$ & $-0.0140(17)$ \\
N1 & $0.045(2)$ & $0.0307(18)$ & $0.050(2)$ & $0.0140(17)$ & $-0.0002(17)$ & $-0.0023(15)$ \\
N2 & $0.057(3)$ & $0.040(2)$ & $0.059(2)$ & $0.021(2)$ & $-0.0016(19)$ & $-0.0045(18)$ \\
C7 & $0.040(2)$ & $0.051(3)$ & $0.051(3)$ & $0.024(2)$ & $0.005(2)$ & $0.006(2)$ \\
C1 & $0.036(2)$ & $0.040(2)$ & $0.046(2)$ & $0.017(2)$ & $0.0036(18)$ & $0.0010(19)$ \\
O3 & $0.047(2)$ & $0.058(2)$ & $0.100(3)$ & $0.0173(17)$ & $-0.0097(18)$ & $-0.0190(18)$ \\
C6 & $0.046(3)$ & $0.035(2)$ & $0.058(3)$ & $0.014(2)$ & $0.005(2)$ & $-0.002(2)$ \\
C4 & $0.058(3)$ & $0.049(3)$ & $0.061(3)$ & $0.034(2)$ & $0.004(2)$ & $0.001(2)$ \\
C2 & $0.039(2)$ & $0.044(3)$ & $0.051(3)$ & $0.019(2)$ & $-0.0017(19)$ & $-0.0007(19)$ \\
C3 & $0.043(3)$ & $0.035(2)$ & $0.051(3)$ & $0.020(2)$ & $-0.0026(19)$ & $0.0000(19)$ \\
C13 & $0.047(3)$ & $0.043(3)$ & $0.048(3)$ & $0.021(2)$ & $0.008(2)$ & $0.008(2)$ \\
C10 & $0.051(3)$ & $0.048(3)$ & $0.069(3)$ & $0.027(2)$ & $0.006(2)$ & $0.001(2)$ \\
O2 & $0.081(2)$ & $0.0451(19)$ & $0.111(3)$ & $0.032(2)$ & $0.003(2)$ & $-0.0172(19)$ \\
C11 & $0.065(3)$ & $0.047(3)$ & $0.079(4)$ & $0.033(3)$ & $0.006(3)$ & $0.000(2)$ \\
C5 & $0.041(3)$ & $0.051(3)$ & $0.070(3)$ & $0.022(2)$ & $0.005(2)$ & $0.000(2)$ \\
C9 & $0.049(3)$ & $0.044(3)$ & $0.062(3)$ & $0.020(2)$ & $0.002(2)$ & $0.002(2)$ \\
C8 & $0.044(3)$ & $0.031(2)$ & $0.046(2)$ & $0.014(2)$ & $0.0038(19)$ & $0.0014(18)$ \\
C12 & $0.058(3)$ & $0.035(2)$ & $0.071(3)$ & $0.019(2)$ & $0.001(2)$ & $-0.003(2)$ \\
C15 & $0.046(3)$ & $0.045(3)$ & $0.081(3)$ & $0.010(2)$ & $-0.008(2)$ & $-0.009(2)$ \\
C14 & $0.060(4)$ & $0.059(4)$ & $0.135(7)$ & $0.028(3)$ & $0.014(4)$ & $0.006(4)$ \\
F1A & $0.095(10)$ & $0.15(2)$ & $0.23(3)$ & $0.094(15)$ & $0.062(17)$ & $0.12(2)$ \\
F2A & $0.069(11)$ & $0.075(12)$ & $0.33(5)$ & $0.041(9)$ & $-0.09(2)$ & $-0.032(18)$ \\
F3A & $0.137(12)$ & $0.20(2)$ & $0.152(13)$ & $0.107(15)$ & $0.075(9)$ & $0.028(14)$ \\
F1B & $0.078(6)$ & $0.094(10)$ & $0.26(2)$ & $0.058(7)$ & $-0.002(10)$ & $-0.056(12)$ \\
F2B & $0.132(9)$ & $0.257(19)$ & $0.150(9)$ & $0.149(12)$ & $-0.068(7)$ & $-0.062(10)$ \\
F3B & $0.060(7)$ & $0.130(11)$ & $0.194(15)$ & $0.047(7)$ & $0.047(8)$ & $0.054(12)$ \\
& & & & & & \\
\hline
\end{tabular}

Geometric parameters $\left(\AA,{ }^{o}\right)$

\begin{tabular}{llll}
\hline $\mathrm{O} 4-\mathrm{C} 13$ & $1.359(5)$ & $\mathrm{C} 13-\mathrm{C} 8$ & $1.405(5)$ \\
$\mathrm{O} 4-\mathrm{C} 15$ & $1.439(4)$ & $\mathrm{C} 10-\mathrm{C} 9$ & $1.376(5)$ \\
$\mathrm{O} 1-\mathrm{C} 6$ & $1.299(4)$ & $\mathrm{C} 10-\mathrm{C} 11$ & $1.380(6)$ \\
$\mathrm{O} 1-\mathrm{H} 1$ & 0.8200 & $\mathrm{C} 10-\mathrm{C} 14$ & $1.478(7)$ \\
$\mathrm{N} 1-\mathrm{C} 7$ & $1.295(5)$ & $\mathrm{C} 11-\mathrm{C} 12$ & $1.389(6)$ \\
$\mathrm{N} 1-\mathrm{C} 8$ & $1.426(5)$ & $\mathrm{C} 11-\mathrm{H} 11$ & 0.9300 \\
$\mathrm{~N} 2-\mathrm{O} 3$ & $1.227(4)$ & $\mathrm{C} 5-\mathrm{H} 5$ & 0.9300 \\
$\mathrm{~N} 2-\mathrm{O} 2$ & $1.230(4)$ & $\mathrm{C} 9-\mathrm{C} 8$ & $1.378(5)$
\end{tabular}




\begin{tabular}{|c|c|c|c|}
\hline $\mathrm{N} 2-\mathrm{C} 3$ & $1.443(5)$ & C9-H9 & 0.9300 \\
\hline $\mathrm{C} 7-\mathrm{C} 1$ & $1.428(5)$ & $\mathrm{C} 12-\mathrm{H} 12$ & 0.9300 \\
\hline $\mathrm{C} 7-\mathrm{H} 7$ & 0.9300 & $\mathrm{C} 15-\mathrm{H} 15 \mathrm{~A}$ & 0.9600 \\
\hline $\mathrm{C} 1-\mathrm{C} 2$ & $1.402(5)$ & C15-H15B & 0.9600 \\
\hline $\mathrm{C} 1-\mathrm{C} 6$ & $1.428(5)$ & $\mathrm{C} 15-\mathrm{H} 15 \mathrm{C}$ & 0.9600 \\
\hline $\mathrm{C} 6-\mathrm{C} 5$ & $1.411(5)$ & $\mathrm{C} 14-\mathrm{F} 1 \mathrm{~A}$ & $1.225(17)$ \\
\hline $\mathrm{C} 4-\mathrm{C} 5$ & $1.360(6)$ & $\mathrm{C} 14-\mathrm{F} 2 \mathrm{~A}$ & $1.228(19)$ \\
\hline $\mathrm{C} 4-\mathrm{C} 3$ & $1.397(5)$ & $\mathrm{C} 14-\mathrm{F} 3 \mathrm{~A}$ & $1.481(17)$ \\
\hline $\mathrm{C} 4-\mathrm{H} 4$ & 0.9300 & $\mathrm{C} 14-\mathrm{F} 1 \mathrm{~B}$ & $1.267(12)$ \\
\hline $\mathrm{C} 2-\mathrm{C} 3$ & $1.368(5)$ & $\mathrm{C} 14-\mathrm{F} 3 \mathrm{~B}$ & $1.295(13)$ \\
\hline $\mathrm{C} 2-\mathrm{H} 2$ & 0.9300 & $\mathrm{C} 14-\mathrm{F} 2 \mathrm{~B}$ & $1.379(12)$ \\
\hline $\mathrm{C} 13-\mathrm{C} 12$ & $1.383(5)$ & & \\
\hline $\mathrm{C} 13-\mathrm{O} 4-\mathrm{C} 15$ & $117.4(3)$ & $\mathrm{C} 12-\mathrm{C} 11-\mathrm{H} 11$ & 119.5 \\
\hline $\mathrm{C} 6-\mathrm{O} 1-\mathrm{H} 1$ & 109.5 & $\mathrm{C} 4-\mathrm{C} 5-\mathrm{C} 6$ & $121.0(4)$ \\
\hline $\mathrm{C} 7-\mathrm{N} 1-\mathrm{C} 8$ & $124.3(4)$ & $\mathrm{C} 4-\mathrm{C} 5-\mathrm{H} 5$ & 119.5 \\
\hline $\mathrm{O} 3-\mathrm{N} 2-\mathrm{O} 2$ & 122.0 & $\mathrm{C} 6-\mathrm{C} 5-\mathrm{H} 5$ & 119.5 \\
\hline $\mathrm{O} 3-\mathrm{N} 2-\mathrm{C} 3$ & $119.2(4)$ & $\mathrm{C} 10-\mathrm{C} 9-\mathrm{C} 8$ & $120.7(4)$ \\
\hline $\mathrm{O} 2-\mathrm{N} 2-\mathrm{C} 3$ & $118.8(4)$ & $\mathrm{C} 10-\mathrm{C} 9-\mathrm{H} 9$ & 119.6 \\
\hline $\mathrm{N} 1-\mathrm{C} 7-\mathrm{C} 1$ & $121.0(4)$ & $\mathrm{C} 8-\mathrm{C} 9-\mathrm{H} 9$ & 119.6 \\
\hline $\mathrm{N} 1-\mathrm{C} 7-\mathrm{H} 7$ & 119.5 & $\mathrm{C} 9-\mathrm{C} 8-\mathrm{C} 13$ & $119.5(4)$ \\
\hline $\mathrm{C} 1-\mathrm{C} 7-\mathrm{H} 7$ & 119.5 & $\mathrm{C} 9-\mathrm{C} 8-\mathrm{N} 1$ & $124.6(4)$ \\
\hline $\mathrm{C} 2-\mathrm{C} 1-\mathrm{C} 6$ & $119.1(4)$ & $\mathrm{C} 13-\mathrm{C} 8-\mathrm{N} 1$ & $115.8(4)$ \\
\hline $\mathrm{C} 2-\mathrm{C} 1-\mathrm{C} 7$ & $120.0(4)$ & $\mathrm{C} 13-\mathrm{C} 12-\mathrm{C} 11$ & $119.1(4)$ \\
\hline $\mathrm{C} 6-\mathrm{C} 1-\mathrm{C} 7$ & $120.9(4)$ & $\mathrm{C} 13-\mathrm{C} 12-\mathrm{H} 12$ & 120.4 \\
\hline $\mathrm{O} 1-\mathrm{C} 6-\mathrm{C} 5$ & $119.7(4)$ & $\mathrm{C} 11-\mathrm{C} 12-\mathrm{H} 12$ & 120.4 \\
\hline $\mathrm{O} 1-\mathrm{C} 6-\mathrm{C} 1$ & $121.9(4)$ & $\mathrm{O} 4-\mathrm{C} 15-\mathrm{H} 15 \mathrm{~A}$ & 109.5 \\
\hline $\mathrm{C} 5-\mathrm{C} 6-\mathrm{C} 1$ & $118.4(4)$ & $\mathrm{O} 4-\mathrm{C} 15-\mathrm{H} 15 \mathrm{~B}$ & 109.5 \\
\hline $\mathrm{C} 5-\mathrm{C} 4-\mathrm{C} 3$ & $120.3(4)$ & $\mathrm{H} 15 \mathrm{~A}-\mathrm{C} 15-\mathrm{H} 15 \mathrm{~B}$ & 109.5 \\
\hline $\mathrm{C} 5-\mathrm{C} 4-\mathrm{H} 4$ & 119.9 & $\mathrm{O} 4-\mathrm{C} 15-\mathrm{H} 15 \mathrm{C}$ & 109.5 \\
\hline $\mathrm{C} 3-\mathrm{C} 4-\mathrm{H} 4$ & 119.9 & $\mathrm{H} 15 \mathrm{~A}-\mathrm{C} 15-\mathrm{H} 15 \mathrm{C}$ & 109.5 \\
\hline $\mathrm{C} 3-\mathrm{C} 2-\mathrm{C} 1$ & $120.5(4)$ & $\mathrm{H} 15 \mathrm{~B}-\mathrm{C} 15-\mathrm{H} 15 \mathrm{C}$ & 109.5 \\
\hline $\mathrm{C} 3-\mathrm{C} 2-\mathrm{H} 2$ & 119.8 & $\mathrm{~F} 1 \mathrm{~A}-\mathrm{C} 14-\mathrm{F} 2 \mathrm{~A}$ & $111.5(16)$ \\
\hline $\mathrm{C} 1-\mathrm{C} 2-\mathrm{H} 2$ & 119.8 & $\mathrm{~F} 1 \mathrm{~A}-\mathrm{C} 14-\mathrm{F} 3 \mathrm{~A}$ & $100.7(13)$ \\
\hline $\mathrm{C} 2-\mathrm{C} 3-\mathrm{C} 4$ & $120.7(4)$ & $\mathrm{F} 2 \mathrm{~A}-\mathrm{C} 14-\mathrm{F} 3 \mathrm{~A}$ & $100.8(18)$ \\
\hline $\mathrm{C} 2-\mathrm{C} 3-\mathrm{N} 2$ & $119.8(4)$ & $\mathrm{F} 1 \mathrm{~B}-\mathrm{C} 14-\mathrm{F} 3 \mathrm{~B}$ & $110.7(11)$ \\
\hline $\mathrm{C} 4-\mathrm{C} 3-\mathrm{N} 2$ & $119.5(4)$ & $\mathrm{F} 1 \mathrm{~B}-\mathrm{C} 14-\mathrm{F} 2 \mathrm{~B}$ & $103.7(10)$ \\
\hline $\mathrm{O} 4-\mathrm{C} 13-\mathrm{C} 12$ & $124.8(4)$ & $\mathrm{F} 3 \mathrm{~B}-\mathrm{C} 14-\mathrm{F} 2 \mathrm{~B}$ & $102.1(10)$ \\
\hline $\mathrm{O} 4-\mathrm{C} 13-\mathrm{C} 8$ & $115.2(4)$ & $\mathrm{F} 1 \mathrm{~A}-\mathrm{C} 14-\mathrm{C} 10$ & $115.9(11)$ \\
\hline $\mathrm{C} 12-\mathrm{C} 13-\mathrm{C} 8$ & $120.0(4)$ & $\mathrm{F} 2 \mathrm{~A}-\mathrm{C} 14-\mathrm{C} 10$ & $117.9(11)$ \\
\hline $\mathrm{C} 9-\mathrm{C} 10-\mathrm{C} 11$ & $119.6(4)$ & $\mathrm{C} 10-\mathrm{C} 14-\mathrm{F} 3 \mathrm{~A}$ & $107.1(8)$ \\
\hline $\mathrm{C} 9-\mathrm{C} 10-\mathrm{C} 14$ & $120.3(5)$ & $\mathrm{F} 1 \mathrm{~B}-\mathrm{C} 14-\mathrm{C} 10$ & $117.5(8)$ \\
\hline $\mathrm{C} 11-\mathrm{C} 10-\mathrm{C} 14$ & $120.1(5)$ & $\mathrm{F} 3 \mathrm{~B}-\mathrm{C} 14-\mathrm{C} 10$ & $111.3(8)$ \\
\hline $\mathrm{C} 10-\mathrm{C} 11-\mathrm{C} 12$ & $121.0(4)$ & $\mathrm{F} 2 \mathrm{~B}-\mathrm{C} 14-\mathrm{C} 10$ & $110.2(6)$ \\
\hline $\mathrm{C} 10-\mathrm{C} 11-\mathrm{H} 11$ & 119.5 & & \\
\hline $\mathrm{C} 8-\mathrm{N} 1-\mathrm{C} 7-\mathrm{C} 1$ & $-177.0(3)$ & $\mathrm{C} 14-\mathrm{C} 10-\mathrm{C} 9-\mathrm{C} 8$ & $-178.1(5)$ \\
\hline $\mathrm{N} 1-\mathrm{C} 7-\mathrm{C} 1-\mathrm{C} 2$ & $177.7(4)$ & $\mathrm{C} 10-\mathrm{C} 9-\mathrm{C} 8-\mathrm{C} 13$ & $0.6(6)$ \\
\hline
\end{tabular}




$\begin{array}{llll}\mathrm{N} 1-\mathrm{C} 7-\mathrm{C} 1-\mathrm{C} 6 & -0.3(6) & \mathrm{C} 10-\mathrm{C} 9-\mathrm{C} 8-\mathrm{N} 1 & 177.7(4) \\ \mathrm{C} 2-\mathrm{C} 1-\mathrm{C} 6-\mathrm{O} 1 & 180.0(4) & \mathrm{O} 4-\mathrm{C} 13-\mathrm{C} 8-\mathrm{C} 9 & 177.8(4) \\ \mathrm{C} 7-\mathrm{C} 1-\mathrm{C} 6-\mathrm{O} 1 & -1.9(6) & \mathrm{C} 12-\mathrm{C} 13-\mathrm{C} 8-\mathrm{C} 9 & -1.0(6) \\ \mathrm{C} 2-\mathrm{C} 1-\mathrm{C} 6-\mathrm{C} 5 & 0.5(6) & \mathrm{O} 4-\mathrm{C} 13-\mathrm{C} 8-\mathrm{N} 1 & 0.4(5) \\ \mathrm{C} 7-\mathrm{C} 1-\mathrm{C} 6-\mathrm{C} 5 & 178.5(4) & \mathrm{C} 12-\mathrm{C} 13-\mathrm{C} 8-\mathrm{N} 1 & -178.4(3) \\ \mathrm{C} 6-\mathrm{C} 1-\mathrm{C} 2-\mathrm{C} 3 & 0.0(6) & \mathrm{C} 7-\mathrm{N} 1-\mathrm{C} 8-\mathrm{C} 9 & 3.1(6) \\ \mathrm{C} 7-\mathrm{C} 1-\mathrm{C} 2-\mathrm{C} 3 & -178.1(4) & \mathrm{C} 7-\mathrm{N} 1-\mathrm{C} 8-\mathrm{C} 13 & -179.6(4) \\ \mathrm{C} 1-\mathrm{C} 2-\mathrm{C} 3-\mathrm{C} 4 & -0.3(6) & \mathrm{O} 4-\mathrm{C} 13-\mathrm{C} 12-\mathrm{C} 11 & -177.7(4) \\ \mathrm{C} 1-\mathrm{C} 2-\mathrm{C} 3-\mathrm{N} 2 & 179.8(4) & \mathrm{C} 8-\mathrm{C} 13-\mathrm{C} 12-\mathrm{C} 11 & 0.9(6) \\ \mathrm{C} 5-\mathrm{C} 4-\mathrm{C} 3-\mathrm{C} 2 & 0.2(6) & \mathrm{C} 10-\mathrm{C} 11-\mathrm{C} 12-\mathrm{C} 13 & -0.4(7) \\ \mathrm{C} 5-\mathrm{C} 4-\mathrm{C} 3-\mathrm{N} 2 & -179.9(4) & \mathrm{C} 9-\mathrm{C} 10-\mathrm{C} 14-\mathrm{F} 1 \mathrm{~A} & 136(2) \\ \mathrm{O} 3-\mathrm{N} 2-\mathrm{C} 3-\mathrm{C} 2 & -0.7(6) & \mathrm{C} 11-\mathrm{C} 10-\mathrm{C} 14-\mathrm{F} 1 \mathrm{~A} & -42(2) \\ \mathrm{O} 2-\mathrm{N} 2-\mathrm{C} 3-\mathrm{C} 2 & -177.9(4) & \mathrm{C} 9-\mathrm{C} 10-\mathrm{C} 14-\mathrm{F} 2 \mathrm{~A} & 0(3) \\ \mathrm{O} 3-\mathrm{N} 2-\mathrm{C} 3-\mathrm{C} 4 & 179.4(4) & \mathrm{C} 11-\mathrm{C} 10-\mathrm{C} 14-\mathrm{F} 2 \mathrm{~A} & -178(3) \\ \mathrm{O} 2-\mathrm{N} 2-\mathrm{C} 3-\mathrm{C} 4 & 2.2(6) & \mathrm{C} 9-\mathrm{C} 10-\mathrm{C} 14-\mathrm{F} 1 \mathrm{~B} & -179.0(14) \\ \mathrm{C} 15-\mathrm{O} 4-\mathrm{C} 13-\mathrm{C} 12 & 0.5(6) & \mathrm{C} 11-\mathrm{C} 10-\mathrm{C} 14-\mathrm{F} 1 \mathrm{~B} & 2.9(16) \\ \mathrm{C} 15-\mathrm{O} 4-\mathrm{C} 13-\mathrm{C} 8 & -178.3(4) & \mathrm{C} 9-\mathrm{C} 10-\mathrm{C} 14-\mathrm{F} 3 \mathrm{~B} & -49.9(13) \\ \mathrm{C} 9-\mathrm{C} 10-\mathrm{C} 11-\mathrm{C} 12 & -0.1(7) & \mathrm{C} 11-\mathrm{C} 10-\mathrm{C} 14-\mathrm{F} 3 \mathrm{~B} & 132.0(12) \\ \mathrm{C} 14-\mathrm{C} 10-\mathrm{C} 11-\mathrm{C} 12 & 178.0(5) & \mathrm{C} 9-\mathrm{C} 10-\mathrm{C} 14-\mathrm{F} 2 \mathrm{~B} & 62.5(13) \\ \mathrm{C} 3-\mathrm{C} 4-\mathrm{C} 5-\mathrm{C} 6 & 0.3(6) & \mathrm{C} 11-\mathrm{C} 10-\mathrm{C} 14-\mathrm{F} 2 \mathrm{~B} & -115.5(12) \\ \mathrm{O} 1-\mathrm{C} 6-\mathrm{C} 5-\mathrm{C} 4 & 179.8(4) & \mathrm{C} 9-\mathrm{C} 10-\mathrm{C} 14-\mathrm{F} 3 \mathrm{~A} & -112.9(16) \\ \mathrm{C} 1-\mathrm{C} 6-\mathrm{C} 5-\mathrm{C} 4 & -0.6(6) & \mathrm{C} 11-\mathrm{C} 10-\mathrm{C} 14-\mathrm{F} 3 \mathrm{~A} & 69.1(16) \\ \mathrm{C} 11-\mathrm{C} 10-\mathrm{C} 9-\mathrm{C} 8 & 0.0(7) & & \\ & & & \end{array}$

Hydrogen-bond geometry $\left(A,{ }^{\circ}\right)$

\begin{tabular}{lllll}
\hline$D-\mathrm{H} \cdots A$ & $D-\mathrm{H}$ & $\mathrm{H} \cdots A$ & $D \cdots A$ & $D-\mathrm{H} \cdots A$ \\
\hline $\mathrm{O} 1-\mathrm{H} 1 \cdots \mathrm{N} 1$ & 0.82 & 1.84 & $2.571(4)$ & 148 \\
$\mathrm{O} 1-\mathrm{H} 1 \cdots \mathrm{O} 4$ & 0.82 & 2.76 & $3.468(4)$ & 146 \\
$\mathrm{C} 7-\mathrm{H} 7 \cdots \mathrm{O} 2^{\mathrm{i}}$ & 0.93 & 2.55 & $3.476(7)$ & 176 \\
$\mathrm{C} 9-\mathrm{H} 9 \cdots \mathrm{O} 2^{\mathrm{i}}$ & 0.93 & 2.46 & $3.378(6)$ & 169 \\
\hline
\end{tabular}

Symmetry code: (i) $-y+4 / 3, x-y-1 / 3, z-1 / 3$. 